\title{
Combined venous and arterial thrombosis revealing underlying myeloproliferative disorder in a young patient: a case report
}

Rime Benmalek*, Hanane Mechal, Hatim Zahidi, Karim Mounaouir, Salim Arous, Mohamed El Ghali Benouna, Abdenasser Drighil and Rachida Habbal

\begin{abstract}
Background: Myeloproliferative neoplasms (MPNs) such as polycythemia Vera (PV) and Essential Thrombocythemia (ET) can be associated with a high risk of both venous and arterial thrombosis. However, the co-existence between these two complications is very rare and has never been described before, especially in young adults with no known history of MPNs.

Case presentation: We report the case of a 39 year-old Caucasian Moroccan male patient without cardiovascular risk factors (CVRF), who presented with acute chest pain. He also suffered from a severe headache since 2 weeks. Electrocardiogram (ECG) showed ST segment elevation myocardial infarction in the posterolateral leads. Cerebral Computed Tomography (CT) scan revealed subarachnoid hemorrhage (SAH), and cerebral Magnetic Resonance Angiography (MRA) found a Superior Sagittal Sinus Thrombosis (SSST). Routine blood tests showed raised hemoglobin and hematocrit in addition to leukocytosis and thrombocythemia. His coronary angiography revealed a thrombus in the ostial left circumflex artery (LCX). Further testing revealed positive Janus kinase 2 (JAK2) V617F mutation and low erythropoietin level, confirming the diagnosis of PV according to the 2008 World Health Organization (WHO) criteria. Antithrombotic and anti-ischemic treatments, in addition to myelosuppressive therapy with hydroxyurea, were initiated with a good clinical and biological evolution.
\end{abstract}

Conclusion: This case shows that MPNs are an important cause of thrombosis, especially in young patients with no other risk factors. Early diagnosis and appropriate management are fundamental before the occurrence of life-threatening complications that can sometimes present in unusual forms associating arterial and venous thrombotic events.

Keywords: Myeloproliferative neoplasms, Polycythemia vera, Complications, Acute myocardial infarction, Cerebral venous thrombosis, Young adult

\section{Background}

Myeloproliferative neoplasms (MPNs) are a group of hematological disorders characterized by abnormal proliferation of all hematopoietic bone marrow elements responsible for a state of hyperviscosity and

*Correspondence: Rime.benmalek@gmail.com Cardiology department, Hospital University Center Ibn Rochd, Casablanca, Morocco thrombocytosis. Thromboembolic and cardiovascular events can be life-threatening complications in this disease [1].

Among thrombotic complications, arterial events such as acute myocardial infarction (AMI) and stroke occur in more than two-thirds of cases, often in the setting of atherosclerosis and traditional cardiovascular risk factors (CVRF) [2]. Rarely, arterial thrombotic events affect young adults with no CVRF, and serve as a starting point for revealing one of the less common causes of arterial original author(s) and the source, provide a link to the Creative Commons licence, and indicate if changes were made. The images or other third party material in this article are included in the article's Creative Commons licence, unless indicated otherwise in a credit line to the material. If material is not included in the article's Creative Commons licence and your intended use is not permitted by statutory regulation or exceeds the permitted use, you will need to obtain permission directly from the copyright holder. To view a copy of this licence, visit http://creativecommons.org/licenses/by/4.0/. The Creative Commons Public Domain Dedication waiver (http://creativeco mmons.org/publicdomain/zero/1.0/) applies to the data made available in this article, unless otherwise stated in a credit line to the data. 
thrombosis in young patients : MPNs. Moreover, MPNs are also responsible for venous thrombotic complications in one-third of cases, including cerebral venous thrombosis (CVT) [2].

Nevertheless, the association between these two thrombotic complications is very rare and has never been described before.

This case outlines the importance of pointing out MPNs as an important cause of thrombosis in young patients without CVRF. It emphasizes the necessity of early recognition of the disease, which can sometimes be initially present in unusual forms associating with arterial and venous events to initiate proper management strategies.

\section{Case presentation}

We report the case of a 39 year-old Caucasian Moroccan male patient with no traditional cardiovascular risk factors nor for venous thromboembolism, who presented to the Emergency department with acute retrosternal constrictive chest pain radiating to the left shoulder, that started $14 \mathrm{~h}$ ago, associated with stage II dyspnea according to New York Heart Association (NYHA) classification and with a severe headache that he described as recurrent since 2 weeks, with no associated neurologic deficit nor meningeal syndrome.

Physical examination found a conscious patient with facial erythrosis, a blood pressure of $140 / 75 \mathrm{mmHg}$, a pulse of 103 beats/min (regular), and saturation on room air of $98 \%$ with normal heart, neurological, chest and abdominal examination. The Electrocardiogram (ECG) on admission (Fig. 1) showed ST segment-elevation with a necrosis Q-wave in posterolateral leads (V5-V6, DIaVL, V7-9) and ST depression in V1-4 leads (mirror view). Transthoracic Echocardiography (TEE) revealed hypokinesia of lateral and inferior walls, with a Left Ventricular Ejection Fraction (LVEF) of $40 \%$ and increased filling pressure, in addition to a severe ischemic mitral regurgitation and a moderate tricuspid insufficiency with an estimated transvalvular gradient of $77 \mathrm{mmHg}$, Right ventricular function was normal. The coronary angiography (Fig.2) revealed a thrombus in the ostial Left circumflex artery (LCX) in addition to a short and spastic left main coronary artery (LM), the rest of the arteries were normal with no sign of atherosclerosis.

Given the severe headache not responding to analgesics, a cerebral nonenhanced CT scan was performed, showing blood in the occipital horns of both lateral ventricles with no diffuse SAH (Fisher grade 4).

It was then followed by a Cerebral Magnetic Resonance Angiography (MRA) where a Superior Sagittal Sinus

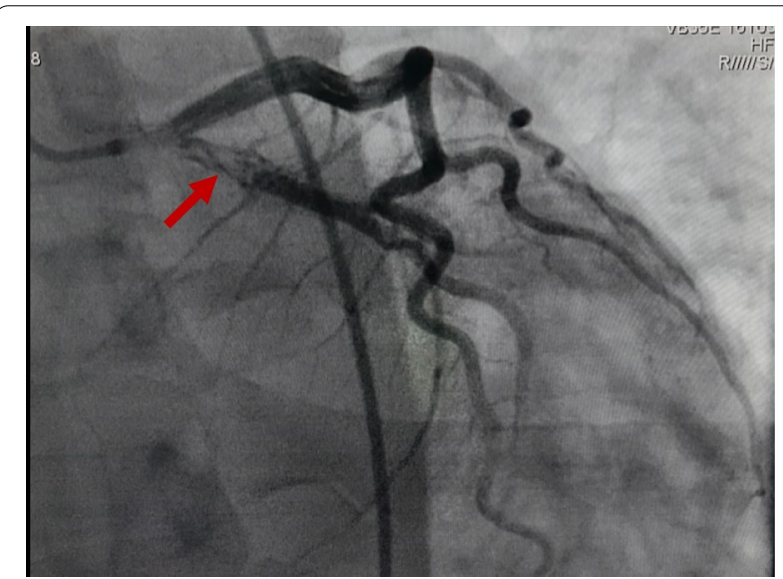

Fig. 2 Coronary angiogram in right anterior oblique (RAO) caudal projection showing a thrombus in the ostial Left circumflex artery (LCX) (Red arrow) in addition to a short and spastic Left main coronary artery (LM).

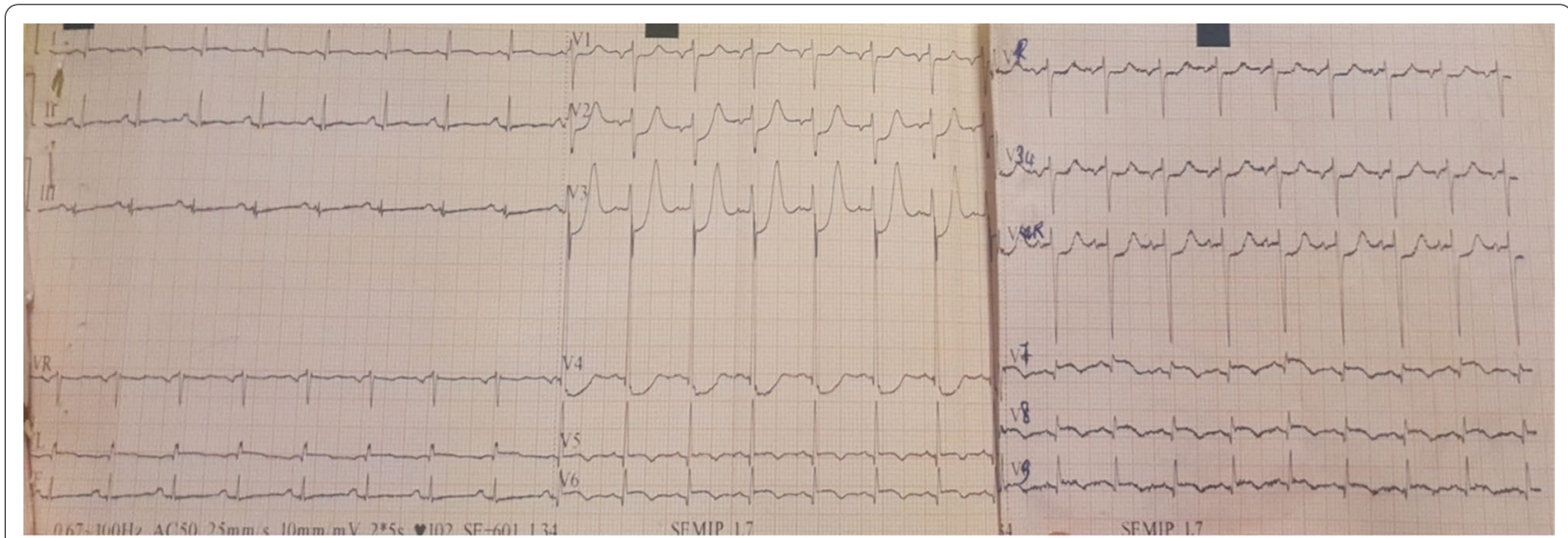

Fig. 1 18-leads Electrocardiogram (ECG) At admission showing ST segment-elevation with a necrosis Q-wave in posterolateral leads with mirror view in anteroseptoapical leads. 

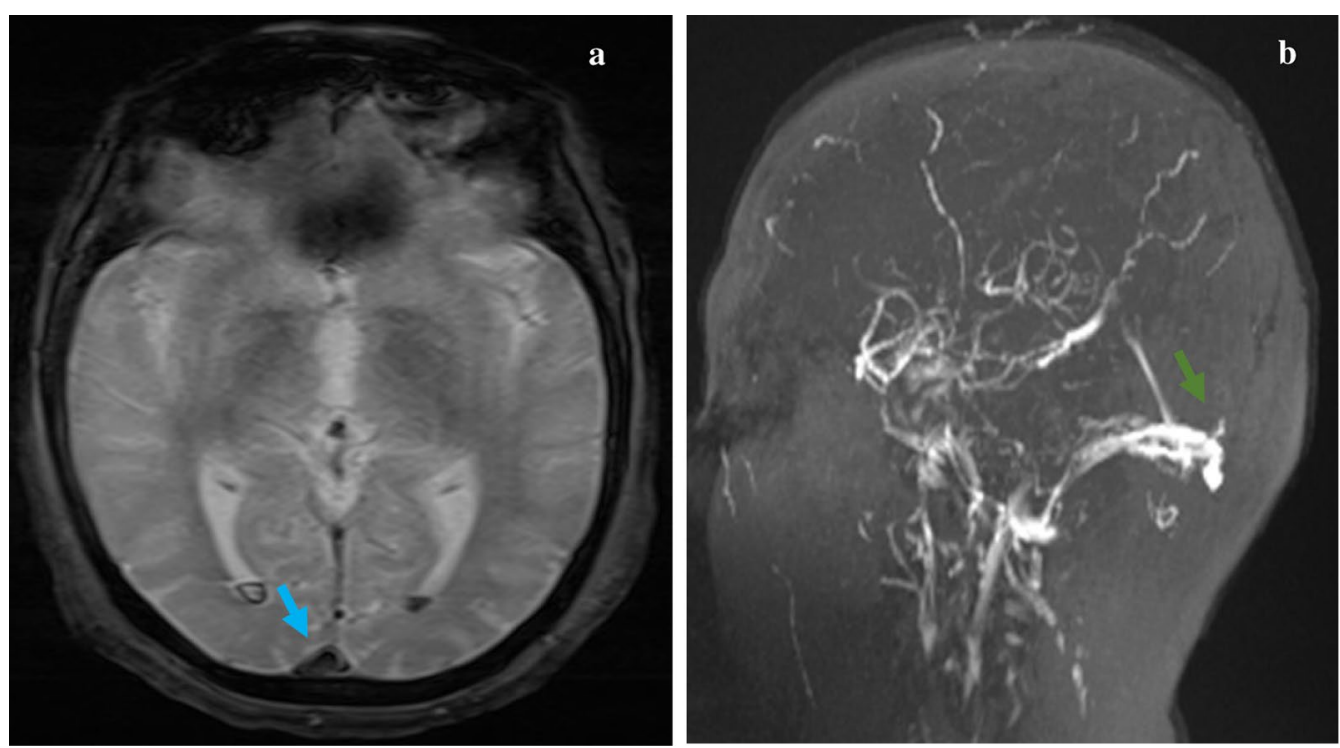

Fig. 3 a Axial T2*GE magnetic resonance imaging showing a low-attenuating thrombus within the superior sagittal sinus with "empty-delta" sign (blue arrow) and hypointense areas in occipital horns of both lateral ventricles corresponding to subarachnoid hemorrhage. b Sagittal magnetic resonance venography showing the filling defect in the superior sagittal sinus indicative of Superior Sagittal Sinus Thrombosis (SSST) (green arrow)

Thrombosis (SSST) was discovered in addition to the Table 1 Laboratory investigation of the patient on admission

\begin{tabular}{lll}
\hline Parameter & Result & Normal range \\
\hline Troponin I & $82 \mathrm{ng} / \mathrm{ml}$ & $<0.1 \mathrm{ng} / \mathrm{ml}$ \\
Aspartate aminotransferase (AST) & $27 \mathrm{U} / \mathrm{L}$ & $5-30 \mathrm{U} / \mathrm{L}$ \\
Alanine aminotransferase (ALT) & $62 \mathrm{U} / \mathrm{L}$ & $5-30 \mathrm{U} / \mathrm{L}$ \\
Lactate dehydrogenase (LDH) & $276 \mathrm{U} / \mathrm{L}$ & $50-150 \mathrm{U} / \mathrm{L}$ \\
Hemoglobin (Hb) & $20.2 \mathrm{~g} / \mathrm{L}$ & $13-16 \mathrm{~g} / \mathrm{L}$ \\
Hematocrit (Ht) & 0.64 & $0.41-0.50$ \\
Red blood cells (RBC) & $7.3 \mathrm{~T} / \mathrm{L}$ & $4.7-6.1 \mathrm{~T} / \mathrm{L}$ \\
White blood cells (WBC) & $22.53 \mathrm{G} / \mathrm{L}$ & $4-10 \mathrm{G} / \mathrm{L}$ \\
Neutrophils & $18.71 \mathrm{G} / \mathrm{L}$ & $2-8 \mathrm{G} / \mathrm{L}$ \\
Platelets & $409 \mathrm{G} / \mathrm{L}$ & $150-400 \mathrm{G} / \mathrm{L}$ \\
Prothrombin time (PT) & $89 \%$ & $70-100 \%$ \\
Fibrinogen & $2.3 \mathrm{~g} / \mathrm{L}$ & $1.8-4 \mathrm{~g} / \mathrm{L}$ \\
Urea & $1.7 \mathrm{mmol} / \mathrm{L}$ & $1.2-3 \mathrm{mmol} / \mathrm{L}$ \\
Creatinin & $1.0 \mathrm{mg} / \mathrm{dL}$ & $0.7-1.3 \mathrm{mg} / \mathrm{dL}$ \\
Na+ & $137 \mathrm{mmol} / \mathrm{L}$ & $135-145 \mathrm{mmol} / \mathrm{L}$ \\
K+ & $4.1 \mathrm{mmol} / \mathrm{L}$ & $3.5-5 \mathrm{mmol} / \mathrm{L}$ \\
Ca++ & $2.2 \mathrm{mmol} / \mathrm{L}$ & $2-2.6 \mathrm{mmol} / \mathrm{L}$ \\
\hline & & \\
\hline
\end{tabular}

intraventricular hemorrhage (Fig. 3).

Baseline Laboratory investigations of the patient are shown in Table 1.
The bone marrow examination showed an increase in all cell lineages, suggestive of a myeloproliferative disorder. Further testing revealed positive JAK2/ V617F mutation and low erythropoietin level $(1.2 \mathrm{mU} /$ $\mathrm{ml}$ ), cytogenetic study for BCR/ABL1 rearrangement was negative. The tests for Antinuclear Antibodies (AAN), antiphospholipid antibodies (APA), anticardiolipin antibody, and lupus anticoagulant were negative. Findings in abdominal ultrasound were unremarkable with no hepatosplenomegaly. Based on these results, the diagnosis of PV was confirmed accordingly to the World Health Organization (WHO) revised criteria (Table 2) [3].

For his myocardial infarction, the patient was first treated with ramipril, bisoprolol, atorvastatin and furosemide in addition to low dose aspirin in concertation with the neurologists, after a repeat cerebral CT scan that showed stationary subarachnoid hemorrhage (SAH) after 48 hours. Cytoreductive therapy was also initiated, starting with $15 \mathrm{mg} / \mathrm{kg}$ per day of hydroxyurea.

Given the patient's high thrombotic risk, and his concomitant cerebral venous thrombosis (CVT), we cautiously started anticoagulation (AC) therapy. We initiated, in close collaboration with the neurology team, low doses of intravenous unfractionated heparin, starting with $18 \mathrm{UI} / \mathrm{Kg} / \mathrm{D}$ with a target partial thromboplastin time (PTT) of 1.5 times the control value.

On the second week, oral AC with Vitamin K Antagonists (VKA) was started, after no evidence of worsening 
Table 2 World Health Organization revised criteria for the diagnosis of Polycytemia Vera [3]

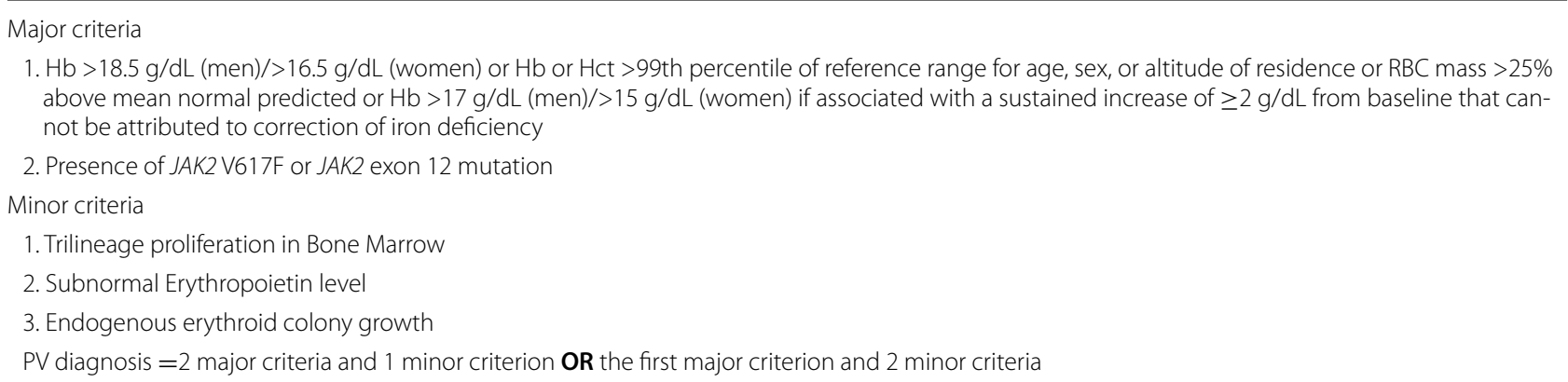

$H c t$ hematocrit, $H b$ hemoglobin, $P V$ polycythemia vera, $R B C$ red blood cell

of SAH, with a lower International Normalized Ratio (INR) target to 2.0-2.5.

The patient's progress was favorable after 3 weeks of treatment and a close neurogical monitoring, with clinical improvement, and progressive correction of hematological parameters. After 21 days, the repeat CT scan showed an important decrease of the SAH secondary to the CVT.

He was discharged from hospital, with dual therapy associating acenocoumarol and low dose aspirin. He was then followed-up as outpatient by a multidisciplinary team of cardiologists, neurologists and hematologists, with a good clinical evolution and a complete regression of his CVT and SAH.

\section{Discussion}

MPNs are a group of hematological disorders, including PV, myelofibrosis (MF) and Essential Thrombocytemia (ET), characterized by clonal stem-cell proliferation of all three lineages [2]. Polycythemia Vera (PV) is a rare disease with an incidence of 2.3 per 100,000 persons per year, with a higher incidence among men of more than 60 years [4], exceptional in younger individuals under 40 years, like our patient. Approximately $96 \%$ of patients with PV have a mutation of the JAK2 gene [2]. The diagnosis of PV is based on the WHO's revised criteria (Table 2) [3].

The disease can be an incidental finding after a routine blood test or present with symptoms mainly related to thrombosis and/or hemorrhage. Indeed, PV is characterized by increased RBC mass resulting in blood hyperviscosity and a higher risk of thrombosis. Pathogenesis of the thrombophilic state acquired in PV is complex and multifactorial and can be summed up in two main mechanisms: The first one is the expression of a prothrombotic phenotype by the abnormal MPN clone-derived blood cells, and the other involves the inflammatory response of host vascular cells that become procoagulant
[1]. Moreover, disease related factors, such as increased blood cell counts, and mostly the presence of the JAK2 mutation, can interact with patient related factors, such as age, history of previous thrombotic events, obesity, hypertension, hyperlipidemia, thrombophilia and other known risk factors for thromboembolism increasing the risk of thrombosis.

In our case, the patient had no history of traditional cardiovascular nor venous thromboembolism risk factor.

Thrombotic complications can be classified into 2 categories : microvascular and macrovascular. Microvascular complications (e.g : Erythromelalgia, headache, visual disturbance, tinnitus...) are the result of thrombus formation in small blood vessels, whereas macrovascular complications are the consequence of thrombi formation in big arteries or veins, and are more serious, accounting for $45 \%$ of deaths in PV patients [5]. Events involving the arterial system represent two third of the events and include ischemic stroke, Acute Myocardial Infarction (AMI), and peripheral arterial thrombosis. Venous thrombotic events (VTE) are not as frequent (one third), and are represented by deep vein thrombosis, pulmonary embolism, intraabdominal, and CVT [6].

These thrombotic events, particularly arterial, can occur before diagnosis and reveal the disease in almost one fifth of the cases, and can be the leading cause of mortality among patients with PV [7]. A retrospective international study of 1545 patients found thrombotic complications responsible for death in $9 \%$ of patients [7], and another study of 1213 patients reported that $30 \%$ of 145 arterial thrombotic events and $11 \%$ of 87 VTE were fatal, causing $24 \%$ and $6 \%$ of all deaths [8].

Our case reports the unique situation of a patient with no family history and no cardiovascular risk factors who experienced both major arterial and venous thrombosis, that eventually revealed PV after a thorough evaluation.

Despite the association of PV with coronary artery disease, its presentation as AMI remains very rare, and the 
association with CVT is exceptional. In the literature, the majority of cases were young males with minimal coronary occlusion, like it was the case for our patient. Rossi et al. studied 149 patients with PV for 10 years and found that $11.4 \%$ developed MI [9]. Benita et al reported the case of a 30-year-old male man who died from AMI as initial manifestation of PV. On autopsy, no atherosclerotic changes nor thrombotic occlusion were found on the coronary arteries, only a marked intimal proliferation leading to multiple occlusions [10].

On the other hand, CVT represent an even rarer complication of PV that can lead to death in up to $8.3 \%$ of patients as reported by Ferro et al [11]. In PV patients, it is often the consequence of raised venous and capillary pressure due to the hyperviscosity state that results in a lower cerebral perfusion, responsible of ischemic injury and cytotoxic edema. Moreover, Thrombosis of cerebral sinuses causes intracranial pressure that can cause parenchymal hemorrhage and vasogenic and cytotoxic edema [12]. Our patient had in addition to his MI, a SSST CVT revealed in the MRA associated to a meningeal hemorrhage. The co-existence between both arterial and venous thrombosis in the same patient was never described before.

Several mechanisms of such major thrombotic events in patients without risk factors are described. Among them, an increased hematocrit> $45 \%$ is associated to an increased risk of cardiovascular events [13], which can be explained in our patient who had an $\mathrm{Ht}$ rate of $64 \%$. Similarly, quantitative and qualitative platelet abnormalities as well as leukocytosis, are at a higher risk of AMI [14], like our patient who had in addition to polycythemia, an important leukocytosis and a slight thrombocythemia. Recently, the JAK2 V617F was found to be an independent risk factor for major vascular events [15], which was also the case in our patient. Finally, other risk factors such as older age, prior history of thrombosis, and cardiovascular risk factors, have been assessed in multiple studies [8].

Generally the treatment of MI secondary to MPNs disorders is a challenge since it requires careful attention to maintain the balance between the hemorrhagic and thrombotic risk. A multidisciplinary management between the cardiologist, interventional cardiologist and the hematologist should be undertook. Percutaneous reperfusion associated with antithrombotic treatment followed by cytoreductive therapy is recommended. Hydroxyurea is suggested as first-line myelosuppressive treatment, and anagrelide is recommended as secondline therapy [16]. As for our patient, coronary angiography showed no critical stenosis requiring reperfusion, however, it revealed a thrombotic lesion of the LCX. Antiplatelet therapy consisting of low dose Aspirin was initiated after stationary SAH in the repeat cerebral CT scan performed at day 2. Considering the patient's concomitant CVT and his high thrombotic risk, we carefully started AC despite his $\mathrm{SAH}$, in concertation with the neurologists, and after performing a repeat $\mathrm{CT}$ brain scan that showed regression of the SAH. In a limited number of studies, AC was found to be beneficial for cases of CVT complicated by SAH. In Rodallec et al review, the authors suggested that $\mathrm{AC}$ was safe, even in patients with cerebral hemorrhage [17]. On the 5th day, we initiated low doses of intravenous unfractionated heparin, starting with $18 \mathrm{UI} / \mathrm{Kg} / \mathrm{D}$ with a target PTT of 1.5 times the control value. On the second week, oral AC with acenocoumarol was started, after no evidence of worsening of $\mathrm{SAH}$, with a lower International Normalized Ratio (INR) target to 2.0-2.5.

The proper time to start AC in such cases was not clearly defined in the literature, and a delay of 4-33 days was observed after the onset of symptoms [18].

In our case, knowing that SAH was secondary to CVT, we started AC early and achieved a good recovery in the patient. After 3 weeks the repeat CT scan showed an important decrease of the SAH. The patient was discharged under dual therapy (acenocoumarol+Aspegic) associated with hydroxyurea, and kept being followedup as outpatient by a multidisciplinary team with a good clinical and neurological progress.

As for the oral anticoagulation, recent studies showed that direct oral anticoagulants (DOACs) such as rivaroxaban and apixaban, can be a good alternative to VKA for the treatment of MPNs-associated VTE. In his review, De Stefano demonstrates the efficacy and safety of DOACs, with less thrombosis recurrence, suggesting that patients with VTE at higher bleeding risk should be considered for treatment with DOACs instead of VKA [19]. These findings were confirmed by a recent cohort study that showed that in MPN patients with VTE, the use of DOACs was associated with very low recurrence rates of VTE and no major bleeding complication [20]. In our patient, VKA were preferred over DOACS for cost and convenience reasons.

Regarding the prognosis, in a retrospective multicenter study of 497 patients, De Stefano et al. studied the recurrence rate of thrombotic events in patients with MPNs who had previously suffered arterial or venous thrombosis or both. They reported that treatment associating antiplatelet and VKA showed effectiveness in preventing recurrence and re-thrombosis of $58 \%$ and $68 \%$ respectively. Their study also showed that the best preventive strategy in these cases is represented by the adjunction of cytoreductive therapy [21]. Another Korean study showed that previous thrombotic history and a positive JAK2 V617F mutation, like in our patient's case, were 
associated to an increased 10-year cumulative rate of thrombohemorrhagic events in patients with MPNs [22].

\section{Conclusion}

Major arterial and/or venous thrombotic events can present as the first manifestation of a more general disease. When such complications occur in a young patient with no risk factors, alternative etiologies should be investigated, among them, MPNs. By describing a unique case of a young patient who presented both AMI and CVT that eventually revealed $\mathrm{PV}$, this report highlights the importance of careful assessment of blood cell count abnormalities in such patients to enable early diagnosis and appropriate multidisciplinary management between cardiologists, neurologists and hematologists, that is essential to reduce the risk of further thrombotic events and prevent morbidity and mortality.

\begin{abstract}
Abbreviations
MPNs: Myeloproliferative neoplasms; PV: Polycythemia Vera; ET: Essential Thrombocytemia; CRVF: Cardiovascular risk factors.; WHO: World Health Organization; ECG: Electrocardiogram; TEE: Transthoracic Echocardiography; CT: Computed Tomography; MRA: Magnetic Resonance Angiography; SSST: Superior Sagittal Sinus Thrombosis; SAH: Subarachnoid hemorrhage; LCX: Left circumflex artery; LM: Left main coronary artery; JAK2: Janus kinase 2; AMI: Acute myocardial infarction; CVT: Cerebral venous thrombosis; VTE: Venous thrombotic events; NYHA: New York Heart Association; RBC: Red blood cells; WBC: White blood cells; AC: Anticoagulation; VKA: Vitamin Kantagonists.
\end{abstract}

\section{Acknowledgements}

Not applicable.

\section{Authors' contributions}

RB participated in the design of the study, acquired data, performed a literature review, and drafted the manuscript. HM participated in the study design and coordination and helped to draft and edit the manuscript. $\mathrm{HZ}$ and KM helped in the patient's management and participated in the acquisition of data. SA participated in the design of the study and edited the manuscript. MEB participated in the design of the study and the patient's management. $A D$ acquired data, participated in the design of the study, and edited the manuscript. RH participated in the design of the study and approved the final version of the manuscript. All authors read and approved the final manuscript.

\section{Funding}

The authors have no funding to declare.

\section{Availability of data and materials}

The published information is available from the corresponding author on reasonable request.

\section{Ethics approval and consent to participate}

This study was conducted in accordance with the fundamental principles of the Declaration of Helsinki.

\section{Consent for publication}

Written informed consent was obtained from the patient for the publication of this case report and any accompanying images. A copy of the written consent is available for review by the Editor-in-Chief of this journal.

\section{Competing interests}

The authors declare that they have no competing interests.
Received: 13 July 2020 Accepted: 18 November 2020

Published online: 16 February 2021

\section{References}

1. Krolla MH, Michaelis LC, Verstovsekc S. Mechanisms of thrombogenesis in polycythemia vera. Blood Rev. 2015;29(4):215-21. https://doi. org/10.1016/j.blre.2014.12.002.

2. Raedler Lisa A. Diagnosis and management of polycythemia vera. Am Health Drug Benefits. 2014;7(7 suppl 3):S36-47.

3. Spivak JL, Silver RT. The revised World Health Organization diagnostic criteria for polycythemia vera, essential thrombocytosis, and primary myelofibrosis: an alternative proposal. Blood. 2008;112:231-9.

4. Polycythemia vera facts. FS13. Leukemia \& Lymphoma Society Web site. www.lls.org/content/nationalcontent/resourcecenter/freeeducationma terials/mpd/pdf/polycythemiavera.pdf. June 2012. Accessed 30 June 2014.

5. Falanga A, Marchetti M. Thrombotic disease in the myeloproliferative neoplasms. Hematology Am Soc Hematol Educ Program. 2012;2012:571-81.

6. Marchioli R, Finazzi G, Landolfi R, Kutti J, Gisslinger H, Patrono C, et al. Vascular and neoplastic risk in a large cohort of patients with polycythemia vera. J Clin Oncol. 2005:23:2224-32.

7. Tefferi A, Rumi E, Finazzi G, Gisslinger $H$, Vannucchi AM, Rodeghiero F, et al. Survival and prognosis among 1545 patients with contemporary polycythemia vera: an international study. Leukemia. 2013;27:1874-81.

8. Gruppo Italiano Studio PolicitemiaPolycythemia vera: the natural history of 1213 patients followed for 20 years. Gruppo Italiano Studio Policitemia. Ann Intern Med, $1995 ; 123,656-664$

9. Rossi C, Randi ML, Zerbinati P, Rinaldi V, Girolami A. Acute coronary disease in essential thrombocythemia and polycythemia vera. J Intern Med. 1998;244(1):49-53.

10. Hermanns B, Handt S, Kindler J, Füzesi L. Coronary vasculopathy in polycythemia vera. Pathol Oncol Res. 1998;4(1):37-9.

11. Ferro JM, Canhão P, Stam J, Bousser MG, Barinagarrementeria F. ISCVT Investigators Prognosis of cerebral vein and dural sinus thrombosis: results of the International Study on Cerebral Vein and Dural Sinus Thrombosis (ISCVT). Stroke. 2004;35:664-70.

12. Stam J. Thrombosis of the cerebral veins and sinuses. N Engl J Med. 2005;352:1791-8.

13. Marchioli R, Finazzi G, Specchia G, Cacciola R, Cavazzina R, Cilloni D, et al. Cardiovascular events and intensity of treatment in polycythemia vera. $\mathrm{N}$ Engl J Med. 2013;368:22-33.

14. Landolfi R, Di Gennaro L, Barbui T, De Stefano V, Finazzi G, Marfisi R, et al. Leukocytosis as a major thrombotic risk factor in patients with polycythemia vera. Blood. 2007;109:2446-52.

15. Vannucchi AM, Antonioli E, Guglielmelli P. Influence of the JAK2V617F mutational load at diagnosis on major clinical aspects in patients with polycythemia vera. Blood. 2006;108:6a.

16. Tortorella G, Calzolari M, Tieghi A, Muià N, Piccin A, Gugliotta L. Acute coronary syndrome (ACS) in patients with essential thrombocytemia (ET) What is the best treatment. Int J Cardiol. 2016;203:225-7.

17. Rodallec MH, Krainik A, Feydy A, Hélias A, Colombani JM, Jullès MC, Marteau V, Zins M. Cerebral venous thrombosis and multidetector CT angiography: tips and tricks. Radiographics. 2006;26(1):S5-18.

18. Hegazi MO, Sherif A, Sakr MG, Hassanien OA. Anticoagulation for cerebral venous thrombosis with subarachnoid hemorrhage: a case report. Med Princ Pract. 2010;19(1):73-5.

19. De Stefano V, Finazzi G, Barbui T. Antithrombotic therapy for venous thromboembolism in myeloproliferative neoplasms. Blood Cancer J. 2018;8(7):65.

20. Natalia CG, Andrew JD, Karen AB, Donal PM, Deepti HR, et al. Outcomes of patients receiving direct oral anticoagulants for myeloproliferative neoplasm-associated venous thromboembolism in a large tertiary centre in the UK. Br J Haematol. 2020;189:e64-118.

21. De Stefano V, ZaT, Rossi E, et al. Recurrent thrombosis in patients with polycythemia vera and essential thrombocythemia, incidence, risk factors, and effect of treatments. Haematologica. 2008;93:372-80. https:// doi.org/10.3324/haematol.12053. 
22. Lee HS, Park LC, Lee EM, et al. Incidence rates and risk factors for vascular events in patients with essential thrombocythemia: a multicenter study from Korea. Clin Lymphoma Myeloma Leuk. 2012;12:70-5. https://doi. org/10.1016/j.clml.2011.10.002.

\section{Publisher's Note}

Springer Nature remains neutral with regard to jurisdictional claims in published maps and institutional affiliations.
Ready to submit your research? Choose BMC and benefit from:

- fast, convenient online submission

- thorough peer review by experienced researchers in your field

- rapid publication on acceptance

- support for research data, including large and complex data types

- gold Open Access which fosters wider collaboration and increased citations

- maximum visibility for your research: over $100 \mathrm{M}$ website views per year

At BMC, research is always in progress.

Learn more biomedcentral.com/submissions 